\title{
Selbsterfahrungsübungen als Methode der Suchtprävention - den Umgang mit Sucht- und Genussmitteln lernen?
}

Jens Kalke

Peter Raschke

\author{
Exercises of Self-Experience, a Method of Addiction Prevention - Learning \\ how to Handle Addiction and Pleasure Related Substances?
}

\section{Zusammenfassung}

Der vorliegende Beitrag beschäftigt sich mit Selbsterfahrungsübungen als Methode der Suchtprävention. Dieser konzeptionelle Ansatz ist in der Suchtprävention relativ jung und noch nicht sehr weit verbreitet. Es wird erstmalig für diese Art suchtpräventiver Intervention eine Übersicht zum Stand von Praxis und Forschung gegeben. Neben einer typologischen Einordnung werden der theoretische Ansatz (soziale Lerntheorie bzw. die damit verbundene Selbstwirksamkeitstheorie und der wissenschaftliche Kenntnisstand dargestellt. Das Kernstück bildet jedoch die Darstellung von sieben praktischen Beispielen aus dem deutschsprachigen Raum: In ihnen geht es beispielsweise um das Einhalten von Trinkregeln, um Verzichtsübungen oder um Testfahrten unter Alkoholeinfluss.

Über die Effektivität von Selbsterfahrungsübungen kann noch keine abschließende Aussage getroffen werden. Dafür liegen bislang zu wenige Evaluationsstudien aus diesem Bereich vor. Die vorhandenen Untersuchungen sind jedoch viel versprechend und deuten darauf hin, dass diese Art von suchtpräventiver Intervention wirkt und ein vernünftiger Umgang mit Sucht- und Genussmitteln erlernt werden kann.

\section{Schlliisselwörter}

Suchtprävention · Selbsterfahrungsübungen · soziale Lerntheorie ·

Evaluation

\section{Abstract}

The paper considers exercises of self-experience as a method to prevent addiction. The concept is rather new and not yet widespread. This paper is the first overview on the state of practice and research of this type of intervention. In addition to a typological classification, we will present the theoretical basis (social theory of learning and the related theory of self-efficacy) as well as the scientific state of knowledge. We will mainly present seven examples from German speaking areas concerning for instance the observation of drinking rules, exercises of renunciation or test driving under the influence of alcohol. The efficacy of the exercises of self-experiences cannot yet be evaluated conclusively. A sufficient number of evaluation studies is not yet available. But those studies that are available so far are promising and indicate that this type of addiction prevention is efficient and that it is possible to learn how to handle addiction and pleasure related substances in a sensible way.

Key words

Addiction prevention - exercises of selfexperience - social theory of learning · evaluation

Institutsangaben

Institut für Interdisziplinäre Sucht- und Drogenforschung (ISD),

Klinik für Psychiatrie und Psychotherapie des UKE

Korrespondenzadresse

Dr. Jens Kalke · Institut für Interdisziplinäre Sucht- und Drogenforschung (ISD), Klinik für Psychiatrie und Psychotherapie des UKE · Martinistraße 52 ·20246 Hamburg · E-mail: Kalkej@aol.com

Bibliografie

Suchttherapie 2003; 4: 192-196 @ Georg Thieme Verlag Stuttgart · New York · ISSN 1439-9903 


\section{Einleitung}

Im letzten Jahr berichtete der SPIEGEL in einem kurzen Beitrag über „Geschultes Saufen“ für Jugendliche (Spiegel, Nr. 40/2002). In diesem Projekt, das unter dem Motto „Wer es lernen will, muss es ausprobieren“ steht, soll ein vernünftiger Umgang mit Alkohol geübt werden. Was bei unbedarften Lesern und Leserinnen möglicherweise Kopfschütteln auslöst, hat in der Suchtprävention seit einiger Zeit Konjunktur.

Der vorliegende Beitrag beschäftigt sich mit solchen Selbsterfahrungsübungen als Methode der Suchtprävention. Dieser konzeptionelle Ansatz ist in der Suchtprävention relativ jung und noch nicht sehr weit verbreitet. Der Begriff „Selbsterfahrungsübung“ bezieht sich hier nicht in einem weiteren Sinne auf Projekte, in denen die Beteiligten im Rahmen von thematischen Einheiten, Rollenspielen oder Erlebnispädagogik neue Erfahrungen machen, sondern fokussiert ganz konkret auf angeleitete Selbsterlebnisse im Umgang mit Sucht- und Genussmitteln. Diese pädagogisch begleiteten Übungen können den Verzicht, die Reduktion oder den Gebrauch von Substanzen (bzw. Medien) beinhalten und formulieren den Anspruch, eine primär- und/oder sekundärpräventive Wirkung zu entfalten. Die Zielsetzungen können dabei verschieden sein bzw. sich auch gegenseitig ergänzen: die Abstinenz von bestimmten Substanzen, ein gesundheitsbewusstes und kontrolliertes Konsumverhalten, die Aneignung von Risikokompetenz oder die Fähigkeit zu genießen. Häufig verschmelzen in diesen Projekten primär- und sekundärpräventive Ziele zu einem ganzheitlichen Ansatz.

In diesem Beitrag wird erstmalig für diese Art suchtpräventiver Intervention eine Übersicht zum Stand von Praxis und Forschung gegeben. Nach einer typologischen Einordnung werden der theoretische Ansatz und als Kern des Artikels einige praktische Beispiele vorgestellt. Abschließend erfolgt ein kurzer Ausblick, indem gleichzeitig auf den wissenschaftlichen Kenntnisstand eingegangen wird.

\section{Typologische Einordnung}

Selbsterfahrungsübungen zielen auf die Veränderung bzw. Manifestierung individueller Verhaltensweisen ab. Sie sind der Gruppe der verhaltenspräventiven Interventionen zuzuordnen. In diesem Bereich können folgende Programmtypen unterschieden werden [1]:

- Informationen, Wissensvermittlung

- Förderung der Lebenskompetenz

- Standfestigkeitstraining

- Stressmanagement

- $\quad$ alternative Freizeitgestaltung

- Erlebnispädagogik

- Peer-education-Ansätze

- Verzichtsprogramme

Selbsterfahrungsübungen als eigenständiger Programmtyp tauchen in solchen Systematisierungen bisher nicht auf. Dies liegt daran, dass angeleitete Selbsterlebnisse mit Konsumieren und Verzichten - wie schon erwähnt - eher selten sind.
Die bekannten Interventionen, wie der schulische Nichtraucherwettbewerb, wurden bislang Verzichtsprogrammen oder dem Standfestigkeitstraining zugeordnet [1].

Die Abgrenzung von Selbsterfahrungsübungen zu originären sekundärpräventiven Ansätzen, wie z.B. Drug-Checking oder Safer-Use-Tipps, besteht darin, dass sie in einem pädagogischen Setting stattfinden und immer interaktiv sind. Sie sind professionell angeleitet und enden mit einer gemeinsamen Auswertung der gemachten Erfahrungen. Zudem geht es bei ihnen in der Regel nicht nur um Risikominimierung beim Gebrauch bestimmter legaler und illegaler Substanzen, sondern um das individuelle Konsumverhalten insgesamt. Schließlich richten sich Selbsterfahrungsübungen immer an nicht abhängige Personen.

\section{Theoretischer Ansatz}

Selbsterfahrungsübungen basieren - explizit oder implizit - auf der sozialen Lerntheorie bzw. der damit verbundenen Selbstwirksamkeitstheorie von Bandura [2,3], die als empirisch gut untermauert und praxisrelevant gelten. Diese stellen auch die theoretische Grundlage für einige Lebenskompetenz- oder Standfestigkeitsprogramme („resistance self-efficacy“) dar. Danach werden Fähigkeiten und Einstellungen über Lernprozesse erworben; ein funktionales Verhalten kann sozial erlernt werden. ${ }^{1}$ Bandura spricht vom „Lernen am Modell“ durch Beobachtung von Verhalten und dessen Reproduktion und spätere Anwendung (Nachahmung). Im Einzelnen kann sich das darin ausdrücken, dass neue Verhaltensweisen erlernt, Hemmschwellen für bereits vorhandenes Verhalten steigen bzw. sinken oder dass ein bestehendes Verhalten ausgelöst wird.

Dabei können die Individuen feststellen, wie man durch das eigene direkte Verhalten Einfluss auf die Ergebnisse des Handelns hat. Durch diese „Selbstwirksamkeit“ (self-efficacy) wird die individuelle Handlungsautonomie gestärkt [3]. Unter Selbstwirksamkeit wird die subjektive Überzeugung verstanden, schwierige Aufgaben oder Lebensprobleme aufgrund eigener Kompetenzen bewältigen zu können. Dazu gehört beispielsweise das „Kontrollierbarkeitserleben“, d. h. die Überzeugung, Kontrolle über ein Ereignis ausüben zu können [3]. Auch die Gewissheit, einer Versuchung widerstehen zu können, kann als eine spezifische Form der Selbstwirksamkeit betrachtet werden [4]. Die hierfür benötigten Kompetenzen müssen aber erst noch entwickelt werden. Durch „Erfolgserfahrungen“ bei diesen selbstregulativen Zielerreichungsprozessen können dann Verhaltensweisen manifestiert werden.

Pädagogisch-didaktische Programme, die auf dem Ansatz der sozialen Lerntheorie und der Selbstwirksamkeitstheorie fußen, arbeiten zur Verhaltensbeeinflussung häufig mit Rollenspielen (Theaterstücke, Fantasiereisen etc.). In der suchtpräventiven Arbeit können dies auch Verzichtsübungen sein. Daran wird das

\footnotetext{
${ }^{1}$ Natürlich spielt auch die Verhaltenssteuerung durch gesellschaftliche Rahmenbedingungen (beispielsweise strukturelle Präventionsmaßnahmen wie Preis, Werbeeinschränkungen etc.) und durch das soziale Klima eine wichtige Rolle.
} 
Lernen von (neuen) Verhaltensweisen geübt. Eine solche Verhaltensweise kann auch der selbstkontrollierte Umgang mit Suchtund Genussmitteln sein. Denn schädlicher Drogenkonsum ist mitbedingt durch einen Mangel an Selbstkontrolle [5]. „Neben der Konsumfähigkeit lassen sich durch soziale Lernstrategien auch Fähigkeiten zum Konsumverzicht, vermittelt beispielsweise über Selbstwirksamkeitserwartungen hinsichtlich der Bewältigung von Belastungen oder dem Widerstehen sozialen Drucks erwerben“ [6].

\section{Praktische Beispiele}

Im Folgenden sollen einige praktische Beispiele für Selbsterfahrungsübungen vorgestellt werden. Diese kommen bzw. entstammen alle dem deutschsprachigen Raum. Eine Literaturrecherche in der Datenbank der Europäischen Beobachtungsstelle für Drogen und Drogensucht (EDDRA = Exchange on Drug Demand Reduction Action) ergab bei weit über 100 primär- und sekundärpräventiven Maßnahmen drei Treffer für Selbsterfahrungsübungen (B., C., G.). ${ }^{2}$ Dies ist ein deutliches Zeichen dafür, dass diese Art der Intervention in der europäischen Praxis noch wenig verbreitet sein dürfte. Die anderen vier Projekte (A., D., E., F.) entstammen einem geplanten Handbuch der Suchtprävention, das bald erscheinen wird [7].

\section{A. „Initiierte Abstinenz“}

Bei dieser Übung verzichten die Schüler und Schülerinnen in einem zweiwöchigen Zeitraum auf ein Sucht- bzw. Genussmittel oder schränken ihren Konsum stark ein (bzw. stellen eine Verhaltensweise ein oder schränken ihre Ausübung stark ein). Als Verzichtsgegenstände kommen dabei Substanzen wie Süßigkeiten, Alkohol, Zigaretten oder Cannabisprodukte genauso infrage wie Medien, z. B. Computerspiele oder Fernsehen.

Hinter dieser Maßnahme steckt die lerntheoretische Annahme, dass das Erleben eines bewussten Verzichtes im frühen Alter einen eigenverantwortlichen und problembewussten Umgang mit Sucht- und Genussmitteln (Alkohol, Zigaretten, Süßigkeiten etc.) fördert und zu einem entsprechenden Konsumverhalten führt (auch bei Fernsehen und Computerspielen).

Ein besonderes Element dieses Unterrichtsprogramms ist, dass es an die konkreten Erfahrungen der Schüler mit Sucht- und Genussmitteln anknüpft. Zu diesem Zweck bildet eine anonyme Befragung der Schüler einer Klasse den Ausgangspunkt der suchtpräventiven Maßnahme. Die jeweilige klassenspezifische Konsumsituation wird den Schülern anhand von Folien zurückgespiegelt. (Dies ist der grundlegende Bestandteil des suchtpräventiven Rahmenprogramms „Gläserne Schule“ aus SchleswigHolstein.) Durch die Darstellung dieses „Konsumprofils“ werden die Schüler zu den Verzichtsgegenständen geführt, die für sie (bzw. die Klasse) relevant sind und möglicherweise ein Problem darstellen.

\footnotetext{
2 Auch wenn es sich bei EDDRA dem Namen nach um eine Datenbank zu illegalen Drogen handelt, finden sich hier viele Präventionsmaßnahmen zu legalen Suchtmitteln wie Nikotin oder Alkohol.
}

Die Intervention wurde in einem Kontrollgruppendesign im Rahmen einer von der EU geförderten Studie evaluiert [8]. Die Ergebnisse zeigen, dass das Unterrichtsprogramm nicht nur von den Schülern und Lehrern akzeptiert und positiv bewertet wird, sondern dass es bei einem erheblichen Teil der Schüler auch zu einer Konsumreduktion kommt (in dem 6-monatigen Untersuchungszeitraum).

Eine ähnliche Maßnahme wird auch in Salzburg (Österreich) durchgeführt: „Ich bin so frei! 3-Wochen-ohne.“ Der Unterschied zur „Initiierten Abstinenz“ liegt darin, dass bei diesem Programm die Verzichtsphase drei Wochen umfasst, sich die Klasse auf nur eine Substanz bzw. eine Verhaltensweise als Verzichtsgegenstand verständigt und dass die Maßnahme ohne Befragung und Darstellung des Klassenprofils durchgeführt wird. Evaluationsergebnisse zu diesem Projekt liegen nicht vor.

\section{B. „Be Smart - Don't Start“}

Bei diesem Programm handelt es sich um einen Wettbewerb zum Nichtrauchen für Schulklassen. Es ist inzwischen in vielen europäischen Ländern verbreitet. Die Zielgruppe der Maßnahme sind Schüler der Klassenstufen 5 bis 8, d. h. die Altersgruppe der 11- bis 14-Jährigen. Die Zielsetzung des Programms liegt einerseits darin, eine möglichst langfristige Verzögerung bzw. gänzliche Verhinderung des Einstiegs in das Rauchen bei nichtrauchenden Schülern zu erreichen. Andererseits soll die Einstellung des Rauchens bei den Schülern, die bereits mit dem Rauchen experimentieren, so beeinflusst werden, dass sie nicht zu regelmäßigen Rauchern werden.

„Be Smart - Don’t Start“ zielt darauf ab, das Nichtrauchen durch die Chance auf einen Gewinn attraktiv zu machen und als Normverhalten bei den Jugendlichen zu etablieren. Die Erfahrung des bewussten Nichtrauchens soll belohnt werden. Der Ansatz dieses Programms unterscheidet sich von daher von klassischen Interventionskonzepten, in denen häufig die Vermittlung negativer Folgen des Rauchens im Mittelpunkt steht.

Der Wettbewerb wird auf Klassenebene durchgeführt und die Schüler der teilnehmenden Klassen verpflichten sich, ein halbes Jahr lang nicht zu rauchen. Die Klassen, die das Ziel erreichen, nehmen an einer Gewinnziehung teil und können viele attraktive Preise gewinnen.

Die vorliegenden Evaluationen zu dem Programm zeigen, dass der Wettbewerb eine hohe Akzeptanz bei Schülern und Lehrkräften besitzt [9]. Darüber wurde im Rahmen einer Kontrollgruppenstudie mit Messwiederholung überprüft, ob die Kampagne geeignet ist, den Einstieg in das Rauchen bei Jugendlichen zu verzögern. Die Ergebnisse zeigen in der Follow-up-Messung (vor und ein halbes Jahr nach Ende des Wettbewerbs) einen um 5\% geringeren Anstieg neuer Raucher in der Experimentalgruppe im Vergleich zur Kontrollgruppe. Die Ergebnisse geben Hinweise darauf, dass diese Art der Intervention geeignet ist, den Einstieg in das Rauchen bei Jugendlichen zu verhindern bzw. zumindest zu verzögern.

\section{C. „Junge Männer im Straßenverkehr - Voll im Griff!“}

In dem Bundesland Niedersachsen wurde ein Projekt erprobt, mit dem das Risikobewusstsein junger männlicher Erwachsener 
(18 bis 24 Jahre) beim Umgang mit Alkohol am Lenkrad gestärkt werden soll. Das Ziel ist Punktnüchternheit im Straßenverkehr. Dadurch soll erreicht werden, dass die Zahl der alkoholbedingten Unfälle, die bei dieser Zielgruppe überdurchschnittlich hoch ist, gesenkt wird.

Die Teilnehmer an dieser Maßnahme müssen in Begleitung eines Fahrlehrers drei Testfahrten durchführen: eine ohne Alkoholeinfluss, eine nach der ersten Alkoholeinnahme (Promillewert dann zwischen 0,2 und 0,4) und eine dritte Fahrt, nachdem erneut Alkohol getrunken worden ist (Promillewert: 0,5-0,8).

Die Fahrlehrer machen sich zu jeder Testfahrt Notizen. Außerdem werden die Fahrten auf Video aufgenommen. Beide Aufzeichnungen werden dann zusammen mit den Teilnehmern in einem Workshop ausgewertet.

Die Evaluation hat ergeben, dass diese Selbsterfahrungsübung zu einer Sensibilisierung der Teilnehmer in Bezug auf ihren Umgang mit Alkohol führt und positive Effekte auf das weitere Fahrverhalten der Teilnehmer hat (siehe auch die ausführliche Projektbeschreibung in diesem Heft).

\section{D. „Saufen will gelernt sein“}

Dieser spezifische Alkoholbaustein des suchtpräventiven Rahmenprogramms "Gläserne Schule“ aus Schleswig-Holstein zielt darauf ab, die schädlichen Wirkungen des Alkoholgebrauchs bei jungen Menschen zu vermindern [10]. Es wird ein kontrollierter Umgang mit alkoholischen Getränken angestrebt. Das Projekt richtet sich an Jugendliche (in der Regel 15- und 16-Jährige), die schon über Konsumerfahrungen mit Alkohol verfügen bzw. gelegentlich oder regelmäßig Alkohol trinken.

Zu diesem Zwecke formulieren die (konsumerfahrenen) Jugendlichen selbst gesetzte Regeln - so genannte "Selbstaufträge“ - in Bezug auf ihren Umgang mit alkoholischen Getränken, an die sie sich über einen Zeitraum von (zunächst) drei Wochen halten sollen. Dabei können die SchülerInnen zwischen vier verschiedenen „Trinkregeln“ wählen: vom vollständigen Alkoholverzicht über „ich trinke nicht, wenn ich Probleme habe“ oder „ich betrinke mich nicht“ bis hin zu „ich trinke höchstens am Wochenende Alkohol“. Begleitend werden thematische Übungen durchgeführt und Informationen zum Thema Alkohol gegeben. Am Ende der drei Wochen werden die gemachten Erfahrungen gemeinsam reflektiert. Die Intervention ist bislang noch nicht evaluiert worden.

\section{E. „Wer nicht genießt, wird ungenießbar“}

In diesem Projekt aus Südtirol (Italien) geht es darum, Jugendliche zwischen 12 und 16 Jahren zur Genussfähigkeit zu erziehen [11]. Die Zielsetzungen dieses Projekts sind im Einzelnen: Steigerung der Genussfähigkeit, kontrollierter Konsum von Genussmitteln, Anregung zu bewusster Ernährung und gesundem Verhalten.

Das Programm setzt sich aus verschiedenen Elementen zusammen, u. a. die Reflexion des eigenen Gesundheits- und Konsumverhaltens in Form eines Genusstagebuchs, ein Genussparcours, Planung und Vorbereitung eines Büfetts, je zwei Einheiten über gesunde Ernährung, ein Kochstudio, Wasserverkostung (ver- schiedene Leitungs- und Mineralwasserproben), das Mixen von alkoholfreien Cocktails für Partys, ein Nachmittag zum Thema „Häppchen für Partys“, vier Verzichtwochen für Freiwillige mit wöchentlicher Reflexion und abschließendem gemeinsamen Pizzaessen. Die konkreten Inhalte und Methoden werden auf die jeweiligen Ziele der teilnehmenden Klassen abgestimmt. Die interne Auswertung des Programms zeigt eine hohe Akzeptanz der Teilnehmer. Beobachtet wurde ein verändertes Gesundheitsund Konsumverhalten bei einem Teil der Jugendlichen. Eine externe Evaluation des Projekts hat es bislang nicht gegeben.

\section{F. „Risflecting“}

Die Entwicklung von Rausch- und Risikokompetenz ist die Zielsetzung dieses Projekts aus Österreich (www.risflecting.at). Dabei wird vorausgesetzt, dass Rausch- und Grenzerfahrungen für viele Jugendliche ein wichtiger Bestandteil ihrer Lebensqualität sind. In angeleiteten und selbst organisierten Settings tauschen die Teilnehmer - in der Regel sind das 13- bis 15-jährige Jugendliche - ihre Rausch- und Grenzerfahrungen aus, erproben Alternativen und reflektieren Wirkungen und Nebenwirkungen dieser Erlebnisse.

Folgende Selbsterfahrungsübungen werden dabei u.a. durchgeführt: Waldspaziergang mit verschlossenen Augen, DJ-Workshops, Cocktail-Mix-Seminar, „Darkroom-Party“ (Party im Dunkeln mit Bar und Musikanlage) und eine gruppendynamische Reise. Bei der letztgenannten Übung planen die Jugendlichen eine zweitägige Reise, bei der das Ziel erst am Tag der Abreise festgelegt wird. Die Funktion der professionellen Betreuer beschränkt sich auf Beobachtung; sie greifen nur in den Prozess ein, wenn gesetzliche Grenzen überschritten werden.

Die dabei gemachten positiven und negativen Erfahrungen werden unter professioneller Anleitung mit dem Ziel ausgewertet, zukünftig die Wirkungen bestimmter Erlebnisse zu verstärken und bei anderen die Risiken zu minimieren.

Das Projekt ist bislang weder wissenschaftlich begleitet noch evaluiert worden.

\section{G. „Spielzeugfreier Kindergarten“}

Dieses Projekt passt zwar nicht ganz in den Rahmen der vorgenannten Maßnahmen, soll aber trotzdem kurz vorgestellt werden, weil es sich auch hier um Lernen am oder besser: ohne Gegenstand handelt.

Ziel dieses suchtpräventiven Projekts im Kindergarten ist die Lebenskompetenzförderung (www.spielzeugfreierkindergarten.de). Für einen begrenzten Zeitraum von drei Monaten werden Spielzeug und alle Materialien (wie z. B. Papier und Stifte) aus dem Kindergarten entfernt, so dass letztendlich nur noch das Mobiliar vorhanden ist. Dadurch soll für einen begrenzten Zeitraum ein neuer Spielraum, ein neuer Erfahrungsraum geschaffen werden, in dem Kinder ihre eigenen Fähigkeiten, ihren eigenen Rhythmus, ihre eigenen Grenzen und Möglichkeiten erkennen können. Durch die temporäre Entfernung des Spielzeugs erhalten die Kinder die Chance, sich stärker auf Gruppenprozesse einzulassen, verschiedene Positionen in der Gruppe zu erproben und sich in anderen Rollen zu versuchen. 
Das Projekt wurde fachlich extern begleitet. Es gibt inzwischen in Deutschland, aber auch in der Schweiz und Österreich zahlreiche Kindergärten, die das Projekt durchgeführt haben. Im Jahr 1999 wurde dem „Network for Mental Health Promotion for Children up to 6 years" das Projekt in Brüssel präsentiert. Es wurde in die Liste der „effektiven Modellprojekte“ aufgenommen.

\section{Ausblick}

Selbsterfahrungsübungen als Methode der Suchtprävention gewinnen offensichtlich immer mehr an Bedeutung. Über ihre Effektivität kann noch keine abschließende Aussage getroffen werden. Dafür liegen bislang zu wenige Evaluationsstudien und entsprechend empirisch gesicherte Erkenntnisse aus diesem Bereich vor. Die vorhandenen Untersuchungen sind jedoch viel versprechend und deuten darauf hin, dass diese Art von suchtpräventiver Intervention wirkt und ein vernünftiger Umgang mit Sucht- und Genussmitteln erlernt werden kann. Dafür spricht auch die generelle Erkenntnis, dass mit interaktiven Programmen bessere Ergebnisse zu erzielen sind als mit reiner Wissensvermittlung, wie eine kürzlich vorgelegte Metaanalyse über schulische Suchtpräventionsprogramme zeigt [1].

Der Ansatz der Selbstkontrolle und Selbstwirksamkeit hat nicht nur in der Prävention, sondern auch im Bereich der Behandlung suchtkranker Personen in den letzten Jahren an Bedeutung gewonnen. Es gibt hier zunehmend Manuale und Anleitungen für selbstinitiierte Ausstiegsprozesse aus der Sucht bzw. für einen kontrollierten Konsum [12]. Auch Therapieprogramme, die nicht ausschließlich auf die vollständige Abstinenz der Klienten zielen, wie beispielsweise das „10-Schritte-Programm zum selbstständigen Erlernen des kontrolliertes Trinkens", etablieren sich im deutschsprachigen Raum zunehmend [13]. Diese Programme zeigen, dass zahlreiche Alkoholiker in der Lage sind, ein kontrolliertes, mäßiges Konsummuster zu erlernen. Dies kann nur dann erfolgen, wenn das „kontrollierte Trinken“ im Bewusstsein der Betreffenden als reale Möglichkeit existiert - wenn es also Teil ihrer Selbstwirksamkeits- bzw. Kompetenzerwartungen geworden ist.

Experimente und Selbsterfahrungsübungen mit Sucht- und Genussmitteln als suchtpräventive Methode stoßen jedoch dort an ihre Grenzen, wo betäubungsmittelrechtliche Regelungen tangiert sind. So dürften angeleitete Übungen zum Umgang mit
Cannabisprodukten auch weiterhin tabu sein. Nur im Rahmen von Modellversuchen wäre dies denkbar. Beispielsweise hätte das (gescheiterte) Projekt „Cannabis in Apotheken“, mit dem die Drogenmärkte getrennt werden sollten, für einen solchen Zweck genutzt werden können [14]. Trotz dieser Beschränkung auf legale Sucht- und Genussmittel sei abschließend die Prognose gewagt, dass Selbsterfahrungsübungen als Methode der Suchtprävention ihre Zukunft noch vor sich haben.

\section{Literatur}

${ }^{1}$ Trimbos Institute. Making schools a healthier place. Manual on effective school-based drug prevention. Utrecht 2002

2 Bandura A. Sozial-kognitive Lerntheorie. Stuttgart: Klett-Cotta, 1979

${ }^{3}$ Self-efficacy in Changing Societies. In: Bandura A (Hrsg). Cambridge: University Press, 1997

${ }^{4}$ Kutza R. Prozessevaluation des schulischen Lebenskompetenzprogramms ALF zur Primärprävention des Substanzmissbrauches. Dissertation. Marburg, 1998

${ }^{5}$ Silbereisen RK, Kastner P. Entwicklung von Drogengebrauch - Drogengebrauch als Entwicklung? In: Oerter R (Hrsg). Lebensbewältigung im Jugendalter. Edition Psychologie. Weinheim, 1985

${ }^{6}$ Schmidt B. Wie kommt es zum Konsum und Mißbrauch von illegalen Substanzen? In: Freitag M, Hurrelmann K (Hrsg). Illegale Alltagsdrogen: Cannabis, Ecstasy, Speed und LSD im Jugendalter. Weinheim: Juventa, 1999

${ }^{7}$ Kalke J, Raschke P, Lagemann C et al. Handbuch für die Suchtprävention. Programme, Projekte und Maßnahmen aus Deutschland, Österreich und der Schweiz. In diesem Handbuch werden 120 Präventionsprojekte aus Deutschland, Österreich und der Schweiz vorgestellt, die von Praktikern und Wissenschaftlern nach bestimmten Qualitätskriterien zusammengestellt worden sind. Freiburg i.B.: Lambertus (im Druck)

${ }^{8}$ Raschke P, Kalke J. Lernen durch Verzicht. Konzept und Wirkung des suchtpräventiven Unterrichtsprogramms „Gläserne Schule“. Baltmannsweiler: Schneider Verlag Hohengehren, 2002

${ }^{9}$ Hanewinkel R, Wiborg G. Primär- und Sekundärprävention des Rauchens im Jugendalter: Effekte der Kampagne „Be Smart - Don't Start“. Gesundheitswesen 2002; 64: 492 - 498

${ }^{10} \mathrm{LSSH} / \mathrm{KOSS}$, Landestelle gegen die Suchtgefahren/Koordinationsstelle schulische Suchtvorbeugung. Jahresbericht 2002. Kiel, 2003

11 Deutsches Schulamt. Gesundheitsförderung in Kindergarten und Schule. Informationsbroschüre. Bozen, 2002

${ }^{12}$ Klingemann H, Sobell L, Barker J et al. Promoting Self-Change from Problem Substance Use. Practical Implications for Policy, Prevention and Treatment. Dordrecht/Boston/London: Kluwer Academic Publishers, 2001

${ }^{13}$ Körkel J. Kontrolliertes Trinken: Eine Übersicht. Suchttherapie 2002; 4: 87-96

${ }^{14}$ Raschke P, Kalke J. Cannabis in Apotheken. Kontrollierte Abgabe als Heroinprävention. Freiburg i. B.: Lambertus, 1997 